\title{
Neuordnung der ambulanten ärztlichen Vergütung
}

\section{ANKE WALENDZIK}

Dr. rer. medic. Anke Walendzik ist wissenschaftliche Mitarbeiterin am Lehrstuhl für Medizinmanagement von Prof. Dr. Jürgen Wasem in Essen und leitet dort die Arbeitsgruppe „Gesundheitssystem, Gesundheitspolitik und Arzneimittelsteuerung“

\author{
Wesentlicher Bestandteil eines einheitlichen \\ Krankenversicherungssystems in Deutschland ist \\ die Schaffung eines gemeinsamen ambulanten \\ Vergütungssystems. Statt ärztliche Leistungen \\ nach Versicherungszugehörigkeit zu steuern und zu \\ Ungleichbehandlung von Patienten entsprechend \\ ihres Krankenversicherungsstatus anzureizen, soll \\ es eine angemessene, qualitativ hochwertige und \\ wirtschaftliche Versorgung fördern. Eine solche \\ Neuordnung jedoch wirft Fragen auf. Nicht nur nach \\ der Gestaltung der Gebührenordnung, sondern auch \\ nach ihrem Leistungs- und Geltungsumfang, nach \\ Zuweisung von Kompetenzen im neuen System, \\ dem mittelfristigen Fortbestand der Budgetierung \\ sowie der Gestaltung einer Konvergenzphase \\ und möglicher Kompensationszahlungen an \\ die niedergelassene Ärzteschaft aufgrund des \\ Wegfalls von Einkünften über Privatpatienten.
}

\section{Hintergrund}

Das duale Versicherungssystem in Deutschland als letztes in Europa erhaltenes Krankenversicherungssystem mit „Systemkonkurrenz“ steht seit Jahren in der Kritik. Neben der fehlenden Verteilungsgerechtigkeit in der Finanzierung der Krankenversicherung - gerade wohlhabendere und gesündere Personen können sich der Solidarität des Sozialversicherungssystems entziehen - stehen gerade die Auswirkungen der zwei verschiedenen Gebührenordnungen im ambulanten Sektor und ihres Zusammenwirkens im Zentrum der Diskussion. Anreize zur Verteilung der Ressourcen folgen nicht der Morbidität der Patienten, sondern differenzieren über die Höhe der gezahlten Vergütung nach Versicherungszuge- hörigkeit. Dies führt zu einer ungleichen Gestaltung des Zugangs zur ambulanten ärztlichen Versorgung: in der Praxis bedeutet dies zumindest höhere Wartezeiten für GKV-als für PKV-Patienten (Kantar Health 2012). Gleichzeitig differieren die Einnahmen der Arztpraxen auch bei Versorgung der gleichen Morbidität erheblich - abhängig vom Anteil der Privatpatienten. Auch die beiden Vergütungssysteme in sich weisen deutliche Steuerungsprobleme auf. Während die Gebührenordnung für Ärzte (GOÄ) mit ihrer vorrangigen Einzelleistungsvergütung Leistungsausdehnung bis hin zur Überversorgung belohnt, verliert sich das GKV-Vergütungssystem in selbst für Experten nicht mehr vollständig durchschaubarer Intransparenz. Obwohl die Sektorengrenzen zur stationären Versor- 
gung in der Versorgungsrealität immer fließender werden, sind auch die Vergütungssysteme zwischen den Sektoren nicht abgestimmt und setzen deshalb auch hier teilweise unangemessene Anreize. Auf der anderen Seite werden in selektivvertraglichen Modellen neue Vergütungsmodelle erprobt, die die bisherige Zweistufigkeit des kollektiven Vergütungssystems aussetzen und differenziertere Systeme von Pauschalen mit einem kleineren Strauß an Einzelleistungsvergütungen kombinieren. Aber auch hier dominieren noch rein ambulante Modelle.

Die Einführung eines einheitlichen Krankenversicherungssystems birgt die Chance der Neugestaltung des damit auch einheitlich gestalteten (kollektiven) ambulanten Vergütungssystems.

vergüten. Für Arztpraxen und Krankenkassen spielen auch die Kalkulierbarkeit der Einnahmen und die Transparenz des zugrundeliegenden Abrechnungssystems eine wichtige Rolle. Immer relevanter wird eine sektorenübergreifende Perspektive bzw. die Harmonisierung von ambulantem und stationärem Vergütungssystem, um Fehlsteuerungen an den Sektorenübergängen zu vermeiden.

\section{Status quo: ambulante Vergütungssysteme in GKV und PKV}

Während oben bereits negative Effekte des Zusammenwirkens von GKV- und PKV-Vergütungssystem im ambulanten Bereich angesprochen wurden, können auch die beiden Ver-

Die Einführung eines einheitlichen Krankenversicherungssystems birgt die Chance der Neugestaltung des ambulanten Vergütungssystems.

Verfolgte Ziele und verwendete Vergütungsformen können besser abgestimmt werden. Aus selektivvertraglichen Erfahrungen kann gelernt werden; gleichzeitig muss als Grundlage für die Weiterentwicklung selektivvertraglicher Wettbewerbsmodelle das Bereinigungsproblem der kollektiven Vergütung endlich sachgerecht gelöst werden.

\section{Ziele für ein ambulantes ärztliches Vergütungssystem}

Wesentliches Ziel eines ärztlichen Vergütungssystems muss die Orientierung am Bedarf der Patienten sein, d.h. in erster Linie an seiner Morbidität. Dabei sollen Anreize zu einer angemessenen, qualitativ hochwertigen und wirtschaftlichen Versorgung gesetzt werden. Gleichzeitig sollen die Leistungserbringer leistungsgerecht vergütet werden. Was das in der Höhe und im Verhältnis zu den Vergütungen anderer professioneller Tätigkeit, sowohl innerhalb als außerhalb des Gesundheitssystems, bedeutet, wird immer einer gesellschaftlichen Diskussion unterliegen. Eine Mindestbedingung für Leistungsgerechtigkeit im Kollektivsystem kann jedoch darin gesehen werden, gleiche Leistung auch gleich zu gütungssysteme in sich einer kritischen Betrachtung unterzogen werden. Dabei ist es im Bereich der Versorgung von GKV-Versicherten auch wichtig, Anregungen aus Vergütungsmodellen der selektivvertraglichen Versorgung aufzugreifen.

\subsection{Die PKV und die Gebührenordnung für Ärzte}

Die ambulante Vergütung der PKVPatienten wie auch anderer „Privatpatienten“ erfolgt nach der per Rechtsverordnung erlassenen Gebührenordnung für Ärzte (GOÄ) in direktem Zahlungsverkehr mit dem Patienten aufgrund des Kostenerstattungsprinzips in der PKV. Die GOÄ beruht dabei im Wesentlichen auf Einzelleistungsvergütungen, denen Punktzahlen zugeordnet sind. Deren Monetarisierung erfolgt über einen fixen Punktwert und einen innerhalb einer Gebührenspanne variablen Aufschlagfaktor. Da die letzte umfassende Anpassung dieser Gebührenordnung auf das Jahr 1996 zurückgeht, bedienen sich die Arztpraxen so genannter Analogleistungen, um den medizinischtechnischen Fortschritt abzubilden. Das Vorherrschen der Einzelleistungsvergütung, dabei im Wesentlichen ohne Qualitätssicherung auf der Ebene von Prozess- und Ergebnisqualität, setzt Anreize zur Leistungsausdehnung und Überversorgung - ein Problem auch für die finanzierenden PKV-Unternehmen. Diese treten basierend auf dem Kosten- erstattungsprinzip zudem grundsätzlich ja in keine direkten Vertragsverhältnisse mit den Leistungserbringern und verfügen deshalb kaum über Steuerungsinstrumente. Die gegenläufigen Interessen einerseits der PKV-Unternehmen an einer Begrenzung der Leistungssteigerungen und andererseits der Ärzteschaft an Erhalt und Ausbau ihrer Einnahmequellen schlagen sich im bisherigen Scheitern der ursprünglich für diese Legislaturperiode geplanten Novellierung und damit Modernisierung der GOÄ nieder.

\subsection{Ambulante ärztliche Vergütung in der GKV im Rahmen des Kollektivvertrags}

Im kollektivvertraglichen Bereich liegt als Gebührenordnung der Vergütung der GKV-Versicherten der Einheitliche Bewertungsmaßstab (EBM) zugrunde, der wie die GOÄ den Gebührenordnungspositionen Punktzahlen zuweist. Ausnahme sind einige so genannte Euro-Leistungen wie z.B. Laborleistungen, die direkt in Euro vermerkt sind. Im Gegensatz zur GOÄ beinhaltet der EBM auch grob altersadjustierte Fallpauschalen, ergänzt durch Komplexpauschalen und Einzelleistungsvergütungen, nutzt also ein breiteres Spektrum von Vergütungsformen. Der Grad der Pauschalierung ist in verschiedenen Arztgruppen unterschiedlich hoch und besonders bei den Hausärzten fortgeschritten. Durch die Budgetierung der - seit 2009 im Grundsatz morbiditätsorientiert fortgeschriebenen - Gesamtvergütungen entsteht ein zweistufiges Vergütungssystem: die Honorarverteilung der Kassenärztlichen Vereinigungen (KVen) gleicht Mengenüberschreitungen über den in den Gesamtvergütungen vereinbarten Leistungsbedarf hinaus per Verteilung der Honorarvolumen an Arztgruppen und Einzelpraxen aus. Dabei bedient sie sich inzwischen, nach Aufhebung der Verpflichtung der Bildung von so genannten Regelleistungsvolumen, verschiedener Systeme der Kürzung des real ausgezahlten Punktwertes, also des Preises der Leistungen. Während der EBM im Bewertungsausschuss zumindest in Teilen auf Basis betriebswirtschaftlicher empirisch basierter Kalkulationen beschlossen und ständig aktualisiert wird, liegt die Honorarverteilung im Wesentlichen im Kompetenzbereich der KVen. Diese müssen sich seit dem GKV- 
Versorgungsstrukturgesetz nur ins Benehmen mit den Krankenkassenvertretungen setzen, so dass von Kassenseite auf dieser Ebene kaum steuernde Maßnahmen vorgenommen werden können. Grundsätzlich erschwert eine so gestaltete Honorarverteilung deutlich, über die Gestaltung der Gebührenordnungspositionen Einfluss auf die Versorgung zu nehmen. Das Gesamtsystem hat zudem einen Grad an Komplexität erreicht, das es nur noch - und das auch meist nur teilweise ausgewiesenen Experten verständlich ist. Für den einzelnen Praxisinhaber ist die Transparenz stark in Frage gestellt.

Eine Morbiditätsorientierung des GKV-Systems findet im Bereich des EBM im Wesentlichen durch einfache Altersadjustierung der Grundpauschalen der jeweiligen Arztgruppen statt, auf der Ebene der Gesamtvergütung jedoch lediglich über die jährliche Veränderungsrate.

Die Komplexität des Systems und seine im Laufe seiner Weiterentwicklung entstandenen inneren Widersprüche erschweren zusätzlich die sachgerechte Bereinigung der Gesamtvergütungen und insbesondere der Regelleistungsvolumen oder Budgets der einzelnen Praxen um selektivvertraglich erbrachte Leistungen. Hierdurch wird die Auflösung von Interessensgegensätzen zwischen den kollektivvertraglich orientierten Vertretern der Leistungserbringer und selektivvertraglich engagierten Kassen und Verbänden stark erschwert. Eine sachgerechte Bereinigung ist jedoch die Voraussetzung für funktionierenden und fairen Wettbewerb zwischen selektivvertraglichen und kollektivvertraglichen Gestaltungsmodellen der Versorgung.

Dem EBM mangelt es ebenfalls an Qualitätsanreizen, die über Anforderungen im Rahmen der Strukturqualität hinausgehen. Gleichzeitig fehlt bisher eine Harmonisierung der Vergütungen zwischen ambulantem und stationärem Sektor, die gerade angesichts der Einführung der spezialfachärztlichen Versorgung immer wichtiger wird.

Die durchschnittliche Höhe der Vergütungen für gleiche Leistungen ist zwar niedriger als im Rahmen der GOÄ, jedoch zeigt auch die ambulante GKV-Vergütung den PKV-Vergütungen vergleichbare Steigerungsraten (Verband der privaten Krankenversicherung 2012). Hier resultiert die Dynamik allerdings nicht wie bei der GOÄ aus dem vorwiegenden Einsatz von Einzelleistungsvergütungen, sondern wesentlich aus gesundheitspolitischen Entscheidungen und Verhandlungserfolgen der Ärzteseite, die sich in Mengensteigerungen und auch in einer sinkenden Honorarverteilungsquote bzw. deutlich steigenden realisierten Punktwerten ausdrücken.

\subsection{Selektivvertragliche Versorgung in der GKV}

In der selektivvertraglichen Versorgung sind zahlreiche neue Vergütungsansätze erprobt worden. In der Regel handelt es sich um Mischsysteme mit stärkerer Orientierung an Kopf- und Fallpauschalen als im kollektiven ambulanten Vergütungssystem (z.B. in den Hausarztverträgen und den Verträgen der besonderen ambulanten Versorgung der AOK Baden-Württemberg) (AOK BadenWürttemberg 2013). Relativ seltener wird mit Vergütungsformen im Rahmen von Pay-for-Performance im Sinne der Anbindung an Prozess- und Ergebnisqualität experimentiert, in dem Zusammenhang steht eher die Anbindung der Vergütungshöhe an Wirtschaftlichkeitsziele im Vordergrund. Alleine integrierte Versorgungsverträge unter Einbeziehung des ambulanten und stationären Sektors bieten die Möglichkeit zur Erprobung der Vergütungsstrukturen an der Sektorgrenze. Die Evaluation entsprechender Modelle kann zur Erstellung des Instrumentarium für die Entwicklung eines kollektiven einheitlichen ambulanten Vergütungssystem genutzt werden.

\section{Elemente eines gemeinsamen ambulanten Vergütungssystems}

Wie für Gesundheitsreformen im Allgemeinen gilt auch für die Gestaltung eines ambulanten ärztlichen Vergütungssystems: es gibt keinen Königsweg. Schon die verschiedenen zu erreichenden Ziele sind durch Trade-offs gekennzeichnet; die einzelnen Vergütungsformen als Instrumente sind in ihren Anreizwirkungen jeweils mit Vor- und Nachteilen behaftet Es geht also um eine möglichst passgenaue Verknüpfung von Vergütungsformen in Hinblick auf die verfolgten Ziele.

\subsection{Grundaufbau des Vergütungssystems}

In Hinblick sowohl auf die Vermeidung von Überversorgung als auch auf eine
Steuerung der Versorgung entsprechend der Morbidität der Versicherten erscheinen stärker morbiditätsorientierte Pauschalen als eine sinnhafte Grundform. In der fachärztlichen Versorgung eignen sie sich auch am besten zur Harmonisierung mit dem DRG-System. Zur Ausdifferenzierung und zum Zweck der Vermeidung von Risikoselektion und Unterversorgung von speziellen Patientengruppen mit besonderen Leistungsbedürfnissen werden diese Pauschalen je nach Arztgruppe in unterschiedlichem Umfang durch Einzelleistungen und Komplexpauschalen zu ergänzen sein. Die Gebührenordnungspositionen sollten betriebswirtschaftlich auf gesicherter empirischer Kostenbasis kalkuliert werden. Außerdem ist gerade in einem pauschalenbasierten System eine ausreichende Qualitätssicherung wichtig. Dies kann auf zweierlei Weise sich jeweils ergänzend geschehen: einerseits können für die Abrechnungsfähigkeit Parameter der Struktur- und Prozessqualität als Voraussetzungen gesetzt werden. Andererseits sollte im Bereich der Ergebnis- und/oder mindestens der Prozessqualität an ergänzenden Pay-forPerformance-Elementen gearbeitet werden. Gerade die Einführung geeigneter Indikatoren der Ergebnisqualität wie z.B. der Senkung von Komplikationsraten bei Diabetes wird wohl eher als mittelfristiger Prozess zu realisieren sein, zumal hier Compliance-Unterschiede zwischen verschiedenen Patientengruppen berücksichtigt werden müssten.

\subsection{Leistungs- und Geltungsumfang}

Es stellt sich die Frage nach dem Leistungsumfang des gemeinsamen ambulanten Vergütungssystems. Der Mindestumfang orientiert sich klar an den Leistungen des neuen Versicherungssystems, welche wiederum auf den jetzigen GKV-Leistungen aufsetzen sollten. Jedoch stellt sich die Frage, ob und inwiefern auch weitere ärztliche Leistungen, die bisher nur über die GOÄ abrechenbar waren, einbezogen werden könnten. Die Frage ist auch im Kontext der in zunehmendem Umfang erbrachten „Individuellen Gesundheitsleistungen“ (IGeL) und der uneinheitlichen Qualität der zugänglichen Informationsquellen in diesem Bereich (Fürstenberg, Katzenmeier et al. 2012) zu sehen. Ein ausreichend 
legitimiertes Gremium wie z.B. der Gemeinsame Bundesausschuss (G-BA) könnte beauftragt werden, auf Basis eines von ihm zu entwickelnden Kriterienkatalogs empfehlenswerte Leistungen im Sinne der bisherigen IGeL-Leistungen mit nachweisbarem oder zumindest auf Basis empirischer Erkenntnisse anzunehmendem Patientennutzen vorzunehmen. Die Aufnahme dieser zusätzlichen Gebührenordnungspositionen außerhalb des Leistungsumfangs des gemeinsamen Versicherungssystems müsste von einem systematisch aufgearbeiteten Informationsangebot begleitet werden.

Die Frage nach dem Geltungsumfang dagegen betrifft die Option des niedergelassenen Arztes, bei Patienten des gemeinsamen Versicherungssystems auch eine höhere Vergütung für seine Leistungen verlangen zu können, die diese ggfs. mit einer Zusatzversicherung abdecken könnten. Über diesen Weg jedoch würde die Steuerung der Behandlung nach Morbidität im Kollektivsystem nicht mehr erreicht, denn es entstünden wie im bisherigen dualen Krankenversicherungssystem Anreize zur vorrangigen Behandlung der „Zuzahler“. Insofern sollte die entsprechende Option für Ärzte, die im neuen Versicherungssystem tätig sind, rechtlich ausgeschlossen werden.

\subsection{Institutionelle Ausgestaltung}

Die Ausgestaltung des neuen Vergütungssystems im Rahmen empirischer Kostenerhebung und auf Basis eines betriebswirtschaftlichen Praxisbetriebssystem, wie oben erwähnt, bedarf eines starken zuarbeitenden Instituts. Dieses sollte wegen der anzustrebenden Harmonisierung der ambulanten ärztlichen Vergütung mit dem DRG-System aus dem stationären Bereich Kompetenzen aus beiden Sektoren vereinigen. Ähnlich wie im DRGBereich sollten Kalkulationspraxen rekrutiert werden, um auch im Längsschnitt Kostenstrukturentwicklungen beobachtbar zu machen. Dies würde auch transparentere Grundlagen für Preisverhandlungen im Rahmen des Vergütungssystems schaffen. Aus all diesen Zusammenhängen bietet sich eine Zusammenlegung des Instituts des Bewertungsausschusses (InBA) und des Institut für das Entgeltsystem im Krankenhaus (InEK) an. Die Trägerschaft sollte weiterhin bei einem paritätisch besetzten Gremium liegen, sei es der G-BA oder ein um die Deutsche Krankenhausgesellschaft (DKG) ergänzter Bewertungsausschuss entsprechend den Vorgaben nach $\$ 87$ Abs. 5a für den neuen Versorgungssektor der ambulanten spezialfachärztlichen Versorgung. .

Eine noch stärker als bisherige Ausgestaltung des Vergütungssystems nach der Morbidität der zu versorgenden Versicherten bedarf verlässlicher Kodierung der ärztlichen Diagnosen. Dies gilt unabhängig davon, ob diese nur als Anlässe zur Auslösung von Pauschalen oder im Rahmen einer Budgetierung als Variablen zur Ermittlung von angemessenen Gesamtvergütungen oder zur Verteilung der Gesamtvergütung auf Arztgruppen oder Einzelpraxen verwenden werden sollen. Hierfür sollten die ambulanten Kodierrichtlinien, wie schon in der Vergangenheit geplant und vom Gesetzgeber wieder verworfen, verpflichtend vorgegeben werden.

Auch die Arbeit an Qualitätsindikatoren bedarf der Verstetigung und mittelfristigen Institutionalisierung.

Bleibt es, was zunächst sicher zu erwarten ist, bei einer budgetierten Gesamtvergütung, so ist die Mitsteuerungsfähigkeit der Kassen auch im kollektiven Vergütungssystem zu stärken. Dies sollte geschehen durch das Wiedereinsetzen der Einvernehmensregelung zwischen KVen und Krankenkassen bei der Honorarverteilung, d.h. einer zwingenden Einigung der Vertragspartner mit Schiedslösung.

\subsection{Differenzierung nach Versorgungsaufträgen von Arztgruppen}

Die Ausdifferenzierung der pauschalierten Vergütungsanteile, die Art und Anzahl von Einzelleistungspositionen und Komplexpauschalen sowie der Grad der Harmonisierung mit dem DRG-System sollte vom Versorgungsauftrag der jeweils vergüteten Arztgruppe abhängen. Ein Arbeitskreis im Rahmen der Friedrich-Ebert-Stiftung zu Wettbewerb, Sicherstellung und Honorierung (Bormann, Engelmann et al. 2013) hat einen entsprechenden Vorschlag zur Differenzierung der Struktur der neuen einheitlichen Vergütungsordnung gemacht, der in Tabelle 1 zusammengefasst ist.

Während der allgemeiner gefasste Versorgungsauftrag in der hausärztlichen Versorgung in der Differenzierung der Pauschalen im Wesentlichen Alter und chronische Erkrankungen berück-

Tabelle 1: Aufbau eines einheitlichen ambulanten ärztlichen Vergütungssystems nach Vorschlag des Arbeitskreises zu Wettbewerb, Sicherstellung und Honorierung im Rahmen der Friedrich-Ebert-Stiftung

\begin{tabular}{|c|c|c|c|}
\hline \multirow[b]{2}{*}{ Versorgungsbereich } & \multirow{2}{*}{$\begin{array}{l}\text { Hausärztlicher } \\
\text { Versorgungsbereich }\end{array}$} & \multicolumn{2}{|c|}{ Fachärztlicher Versorgungsbereich } \\
\hline & & $\begin{array}{l}\text { Fachärzte der } \\
\text { Grundversorgung }\end{array}$ & $\begin{array}{l}\text { Fachärzte der hochspeziali- } \\
\text { sierten Versorgung }\end{array}$ \\
\hline $\begin{array}{l}\text { Altersadjustierte } \\
\text { Fallpauschalen }\end{array}$ & Altersadjustierte Fallpauschalen & $\begin{array}{l}\text { Ggfs. altersadjustierte } \\
\text { Fallpauschalen }\end{array}$ & - \\
\hline $\begin{array}{l}\text { Morbiditätsbezogene } \\
\text { Pauschalen }\end{array}$ & $\begin{array}{l}\text { Chronikerpauschalen, gebun- } \\
\text { den an Parameter der Prozess- } \\
\text { und Strukturqualität, Koordina- } \\
\text { tionstätigkeit, ggfs. Zuschläge } \\
\text { für Multimorbidität }\end{array}$ & $\begin{array}{c}\text { Arztgruppenbezogene mor- } \\
\text { biditätsbasierte Pauschalen, } \\
\text { gebunden an Parameter der } \\
\text { Prozess- und Strukturqualität, } \\
\text { wo möglich Harmonisierung } \\
\text { mit DRG-System }\end{array}$ & $\begin{array}{l}\text { Arztgruppenbezogene mor- } \\
\text { biditätsbasierte Pauschalen, } \\
\text { gebunden an Parameter der } \\
\text { Prozess- und Strukturqua- } \\
\text { lität, Harmonisierung mit } \\
\text { DRG-System }\end{array}$ \\
\hline $\begin{array}{l}\text { Sonstige } \\
\text { Vergütungselemente }\end{array}$ & \multicolumn{3}{|c|}{$\begin{array}{l}\text { Einzelleistungsvergütungen und Komplexpauschalen für besonders förderungswürdige } \\
\text { sowie seltene Leistungen }\end{array}$} \\
\hline $\mathrm{P}_{4} \mathrm{P}$ & \multicolumn{3}{|c|}{ Vergütungsanteil P4P, anhand von Parametern der Ergebnis- und der Prozessqualität } \\
\hline
\end{tabular}

Quelle:(Bormann, Engelmann et al. 2013) 
sichtigt, nähert sich die fachärztliche Versorgung in ihrer Struktur stärker dem stationären Vergütungssystem an. Dabei wird unterschieden zwischen einem fachärztlichen Grundversorgungsbereich wie z.B. in der gynäkologischen Versorgung und Fachärzten der hochspezialisierten Versorgung, deren Leistungen zu einem größeren Anteil stationären Leistungen vergleichbar sind. Die Harmonisierung mit dem DRG-System findet also vorrangig dort statt, wo Schnittstellen der Sektoren zu vermuten sind und Fehlanreize vermieden werden sollen. Neben den traditionellen Strukturelementen eines Vergütungssystems, also in unterschiedlichem Umfang differenzierten Fallpauschalen, Komplexpauschalen und Einzelleistungsvergütungen, bezieht der Vorschlag auch eine Implementierung qualitätssichernder Elemente ein. Dies soll sowohl im Rahmen der Festlegung von Leistungsvoraussetzungen als auch durch die Einführung von P4P-Elementen in der neuen Gebührenordnung geschehen..

\subsection{Budgetierung?}

Das ambulante ärztliche Vergütungssystem der GKV ist seit den 1990er Jahren durch Budgetierung gekennzeichnet - als Reaktion auf ein als nicht angemessen angesehenes Mengenwachstum. Als ökonomische Begründung dient insbesondere die Annahme arztinduzierter Nachfrage nach ärztlichen Leistungen. (Schulenburg and Greiner 2000). Arztinduzierte Nachfrage ist auch einer der Erklärungsansätze für die Abhängigkeit der Leistungsmenge je Patient von der Arztdichte.

Von Seiten der Ärzteschaft wird die Budgetierung dagegen abgelehnt. Dabei wurde insbesondere die bis 2008 praktizierte Grundlohnanbindung der Ausgaben auch im ambulanten Bereich kritisiert, da Lohn- und Gehaltssteigerung in keinem Bezug zu Morbiditätsveränderungen in der Bevölkerung und einer dadurch bedingten Veränderung des Leistungsbedarfs stehen. In diesem Zusammenhang wurde in der Honorarreform 2009 die grundsätzliche Morbiditätsorientierung der Gesamtvergütungen eingeführt, jedenfalls was die Ermittlung der jährlichen Steigerungsraten im weiterhin budgetierten System angeht.

Wie oben erwähnt, zeigen in den letzten Jahren beide bisher praktizierten Vergütungssysteme in GKV und PKV ähnliche Steigerungsraten. Im GKVBereich liegt in den letzten Jahren die Steigerung der erbrachten Menge an Leistungen tendenziell unterhalb der Steigerung der Gesamtvergütungen, so dass die realen Preise nach der Honorarverteilung gestiegen sind. Der jährlich vereinbarte Leistungsbedarf liegt jedoch trotzdem noch regelmäßig unter der erbrachten Leistungsmenge.

Es stellt sich die Frage, ob ein wesentlich auf morbiditätsbasierten Pauschalen aufgebautes System in der Lage ist, ähnlich wie in einigen Selektivverträgen, ohne Budgetierung auszukommen. Dies dürfte in der Realität auch davon abhängen, wie sich im Rahmen der konkreten Ausgestaltung das Verhältnis von pauschalierten Vergütungsbestandteilen und Einzelleistungsvergütungen entwickelt und welche Wachstumsdynamik die Einzelleistungen aufweisen werden. In den ersten Jahren der Einführung des neuen gemeinsamen Vergütungssystems wird man auch Mengeneffekte bei den morbiditätsbasierten Pauschalen aufgrund von Rightcoding-Prozessen beobachten müssen. Insofern wird eine mögliche Aufhebung der Budgetierung erst mittelfristig nach der Einführungsphase des neuen Vergütungssystems zu diskutieren sein.

Im Zusammenhang mit der Budgetierung ist auch die Ungleichheit der regio-

\section{Eine mögliche Aufhebung der Budgetierung wird erst mittelfristig nach der Einführungsphase des neuen Vergütungssystems zu diskutieren sein.}

nalen Verteilung der Mittel zu beachten. Budgetierung ist eine, wenn auch wenig treffsichere Möglichkeit, Überversorgung in Regionen mit angenommener arztinduzierter Nachfrage einzudämmen und Mittel für die Förderung unterversorgter Regionen freizusetzen.

Mit einer Aufhebung der Budgetierung allerdings wäre das Bereinigungsproblem für selektivvertragliche Versorgung automatisch gelöst.

\subsection{Konvergenzfragen}

Für den Übergang in ein gemeinsames Versicherungssystem sind unterschiedli- che Szenarien denkbar. Politisch relativ unwahrscheinlich erscheint die Extremvariante des sofortigen Übergangs aller Versicherten in das neue einheitliche Versicherungssystem. Wahrscheinlicher ist ein gleitender Übergang bei Schließen der Neuzugangsmöglichkeit zur PKV. Dieser könnte flankiert werden von einem Optionszeitraum, in dem PKVBestandsversicherte in das gemeinsame Versicherungssystem freiwillig wechseln können. Gleichzeitig wird die Einführung eines neuen ambulanten Vergütungssystems vermutlich sukzessive erfolgen, so dass mit einem doppelten Konvergenzprozess zu rechnen ist.

Schon wegen der fehlenden Aktualität der GOÄ und ihrer einseitigen Konzentration auf Einzelleistungsvergütungen erscheint sie als Gebührenordnung des Übergangs für ein gemeinsames Versicherungssystem nicht geeignet. Würde also im ersten Schritt das GKV-Vergütungssystem mit dem EBM auf die „neuen"Versicherten übertragen bzw. für alle im gemeinsamen Versicherungssystem Versicherten angewendet, so würde dies ohne weitere Kompensation Einnahmen- und Einkommensverluste bei der niedergelassenen Ärzteschaft induzieren. Diese entstünden sowohl zum Zeitpunkt des Systemübergangs (außer in einem Modell der Trennung der Versicherungssysteme ohne Optionszeitraum, da hier zu Beginn alle Versicherten in ihren jeweiligen Systemen verbleiben) als auch in der weiteren zeitlichen Perspektive durch nunmehr fehlende Neuzugänge in der PKV und entgangene Mehrerlöse durch den demografisch bedingten Mehrbedarf der insgesamt entgangenen PKV-Versicherten. Dabei wären unterschiedliche KV-Regionen und einzelne Arztpraxen unterschiedlich betroffen, je nach bisherigem Anteil der Privatpatienten.

Damit stellt sich die Frage nach einer Kompensation des Honorarausfalls für die ambulant tätige Ärzteschaft. Diese könnte damit begründet werden, dass die Investitionsentscheidungen der niedergelassenen Ärzte unter der Annahme der Stabilität des bestehenden Systems getroffen worden sind, insofern also ein Vertrauensschutz angemessen wäre. Ein weiteres Argument wäre die Annahme, dass das bisherige Vergütungsniveau zur Aufrechterhaltung des derzeitigen Ver- 
sorgungsniveaus notwendig sei. Andererseits werden auch andere Gruppen in und außerhalb des Gesundheitswesens nicht regelmäßig für reformbedingte Systemveränderungen entschädigt. Außerdem könnte auch bei Fortbestehen des PKV-Vollversicherungssystems früher oder später die Notwendigkeit entstehen, aus Gründen des Systemerhalts die ambulanten Vergütungsunterschiede abzuschmelzen.

Entsprechend sind auch Teillösungen für eine Kompensation von Einnahmenverslusten denkbar: so kann nur anteilig oder zeitlich begrenzt kompensiert werden. Möglich wäre auch eine zeitlich degressiv ausgestaltete, also im Laufe der Zeit anteilig sinkende Kompensation. Es stellt sich auch die Frage, ob ein freiwilliger Wechsel von Versicherten in einem wie oben beschriebenen Optionszeitraum Kompensationszahlungen auslösen sollte oder dies nur bei Schaffung eines Kontrahierungszwanges in der GKV für alle bisherigen PKV-Versicherten geschehen sollte.

Modellberechnungen zeigen, dass eine volle Kompensation ärztlicher Honorarverluste je nach Übergangsmodell bis zu 4,3 Milliarden Euro im Umstellungsjahr erfordern würde und innerhalb von 20 Jahren auf fast 6 Milliarden Euro anwachsen könnte. In einem Konvergenzmodell des Übergangs mit Optionszeitraum wie oben beschrieben jedoch würde der auszugleichende Vergütungsausfall im Ausgangsjahr noch nicht entstehen, nach einem Jahr je nach Wechselverhalten der bisher PKV-Versicherten um die 1,5 Milliarden Euro betragen und nach 20 Jahren auf gut 3 Milliarden Euro steigen (Wasem, Buchner et al. 2013).

Die Kompensationszahlungen müssten innerhalb der Regionen, aber auch der Arztgruppen und einzelnen Arztpraxen verteilt werden. Auch hier ergibt sich eine Reihe von Varianten. Soll vorrangig das Ziel des Vertrauensschutzes verfolgt werden, so würde sich eine Verteilung nach erlittenen Honorareinbußen der Ärzte anbieten. Bei Anwendung entsprechender regionaler Verteilungsgrundsätze würde die Zusatzvergütung vor allen Dingen in westliche Bundesländer mit einem bisher höheren Anteil an Privatversicherten fließen. Würde das Prinzip auch innerhalb der Honorarverteilung weiterverfolgt, so würden Praxen mit bisher hohem Patientenanteil von nach GOÄ vergüteten Ver- sicherten vorrangig profitieren. Letztlich würde die als unbefriedigend bewertete alte Allokation der Mittel perpetuiert. Insofern bietet sich eine solche Verteilung der Kompensation allenfalls als kurzfristige Übergangslösung an. Mittelfristig dagegen wäre zu entscheiden, ob die bisherige regionale Verteilung der GKVVergütungen beibehalten werden soll. Alternativ könnte man die Zusatzmittel $\mathrm{zu}$ einer stärker morbiditätsorientierten Verteilung der Gesamtvergütungen und

\section{elfristig dagegen wäre zu} entscheiden, ob die bisherige regionale Verteilung der GKV-Vergütungen beibehalten en soll.

ggfs. auch der Arztgruppentöpfe und RLVs/Arztbudgets verwenden, jedenfalls solange und soweit die Budgetierung Bestand hat. In diesem Falle würde man entweder die Kompensationszahlungen selber entsprechend der Morbidität der versorgten Bevölkerung, gemessen über Diagnosen und demographische Faktoren (Alter und Geschlecht), verteilen. Darüber noch hinausgehend könnte man sie einsetzen, um zur versorgten Morbidität proportionale Gesamtvergütungen in den Kassenärztlichen Vereinigungen zu erreichen, also einem schon 2009 diskutierten Aufteilungsmodell folgen (Wasem and Walendzik 2009). Die Nutzung der Kompensationen zur Redistribution zwischen den KV-Regionen könnte mittelfristig im Sinne einer bedarfs- und damit morbiditätsorientierten Versorgung durchaus sinnvoll sein. Allerdings würde eine Zuweisung solcher Entscheidungen an das KV-System dieses wegen der damit verbundenen regionalen Interessensgegensätze stark unter Druck setzen. Insofern wären hier klare Vorgaben des Gesetzgebers hilfreich.

\section{Ausblick}

Ein neues einheitliches kollektives ambulantes Vergütungssystem ist ein konstituierendes Merkmal für ein erfolgreiches gemeinsames Versicherungssystem, also auch im Rahmen einer Bürgerversicherung. Sein Aufbau muss schrittweise erfolgen. Während die Gebührenordnungspositionen für morbiditätsbasierte Pauschalen und ergänzende Komplex- pauschalen und Einzelleistungen relativ zügig geschaffen werden können, wird die Einführung von Elementen der P4P ein mittelfristiger Prozess sein. Dies gilt besonders, da auch Qualitätsparameter für eine sektorenübergreifende Ergebnisqualität anzustreben sind. Eine mögliche Aufhebung der Budgetierung kann erst dann erwogen werden, wenn Erfahrungen mit einem ausgereiften neuen Vergütungssystem vorliegen.

Auch über die Ausgestaltung einer Kompensation für ärztliche Honorarausfälle wird zu diskutieren sein. Sinnvoll ist es, diese zusätzlichen Geldmittel zumindest mittelfristig für eine Allokation von Ressourcen entsprechend der zu versorgenden Morbidität zu nutzen.

1 AOK Baden-Württemberg (2013). „Hausarztzentrierte Versorgung (HZV) der AOK Baden-Württemberg“. from https://www.aokgesundheitspartner.de/bw/ arztundpraxis/hzv/index. html.

2 Bormann, R., D. Engelmann, et al. (2013). Wettbewerb, Sicherstellung, Honorierung : Neuordnung der Versorgung im deutschen Gesundheitswesen ; Positionspapier im Auftrag der Abteilung Wirtschafts- und Sozialpolitik der Friedrich-Ebert-Stiftung

3 Wiso Diskurs. Friedrich-EbertStiftung. Bonn. 4/2013.

4 Fürstenberg, T., C. Katzenmeier, et al. (2012). Untersuchungen zum Informationsangebot zu Individuellen Gesundheitsleistungen (IGeL). I. I. gmbH. Berlin.

5 Kantar Health (2012). Bevölkerungsumfrage BKK 2011; Thema Arztbesuche.

6 Schulenburg, M. G. v. d. and W. Greiner (2000). Gesundheitsökonomik. Tübingen, Mohr Siebeck.

7 Verband der privaten Krankenversicherung (2012). PKV-Zahlenbericht 2011/2012. Berlin.

8 Wasem, J., F. Buchner, et al. (2013). Ambulante ärztliche Vergütung in einem einheitlichen Versicherungssystem. Kompensation ärztlicher Einkommensverluste in der Konvergenz? Baden-Baden, Nomos.

9 Wasem, J. and A. Walendzik (2009). „Weiterentwicklung der Vergütungsreform. Was bedeutet die Morbiditätsorientierung der Gesamtvergütungen?" Gesellschaftspolitische Kommentare 2009(5): 24-19. 